\title{
A Fruit Quality Management System Based On Image Processing
}

\author{
Ms.Rupali S.Jadhav ${ }^{1}$, PROF. S.S.Patil ${ }^{2}$ \\ ${ }^{1}$ T. K. I. E. T., Warananagar, Electronics Department, Maharashtra, India \\ ${ }^{2}$ T. K. I. E. T., Warananagar Electronics Department, Maharashtra, India
}

\begin{abstract}
Nondestructive quality evaluation of fruits is important and very vital for the food and agricultural industry. The fruits in the market should satisfy the consumer preferences. Traditionally grading of fruits is performed primarily by visual inspection using size as a particular quality attribute. Image processing offers solution for automated fruit size grading to provide accurate, reliable, consistent and quantitative information apart from handling large volumes, which may not be achieved by employing human graders. This paper presents a fruit size detecting and grading system based on image processing. The early assessment of fruit quality requires new tools for size and color measurement. After capturing the fruit side view image, some fruit characters is extracted by using detecting algorithms. According to these characters, grading is realized. Experiments show that this embedded grading system has the advantage of high accuracy of grading, high speed and low cost. It will have a good prospect of application in fruit quality detecting and grading areas.
\end{abstract}

Keywords: embedded system, size detecting, fruit grading, image processing

\section{INTRODUCTION}

In order to improving fruits' quality and production efficiency, reduce labor intensity, it is necessary to research nondestructive automatic detection technology. Fruit nondestructive detection is the process of detecting fruits' inside and outside quality without any damage, using some detecting technology to make evaluation according some standard rules. Nowadays, the quality of fruit shape, default, color and size and so on cannot evaluated on line by the traditional methods. With the development of image processing technology and computer software and hardware, it becomes more attractive to detect fruits' quality by using vision detecting technology. At present, most existing fruit quality detecting and grading system have the disadvantage of low efficiency, low speed of grading, high cost and complexity. So it is significant to develop high speed and low cost fruit size detecting and grading system.

Here two choices are provided for grading either by color and size. In first case we are going to sort circular shaped fruits according color and grading is done according to size. The proposed automated classification and grading system is designed to combine three processes such as feature extraction, sorting according to color and grading according to size. Software development is highly important in thiscolor classification system and for finding size of a fruit. The entire system is designed over MATLAB software to inspect the color and size of the fruit. Here grading can be categories into four ways Red small, Red big, Green small, Green big. Work in this paper considered two different fruits apple or tomato and guava having different features like apple or tomato is red and guava is green and system can sort and grade the fruits according to different attributes such as color and size. It mainly contains four parts: the system overview, fruit size detecting and grading, experiments and results, conclusion. The four parts will be introduced as follows

\section{LITERATURE SURVEY}

Hongshe Dang, Jinguo Song, Qin Guo [1] have proposed fruit size detecting and grading system based onimage processing. The system takes ARM9 as main processor and develops the fruits size detecting programusing image processing algorithms on the QT/Embedded platform. Authors in [2] have proposed system which finds size of different fruits and accordingly different fruits can be sorted using fuzzy logic, here author proposed MATLAB for the features extraction and for making GUI. John B. Njoroge. Kazunori Ninomiya. Naoshi Kondo and Hideki Toita [3] have developed an automated grading system using image processing where the focus is on the fruit's internal and external defects. The system consists of six CCD cameras. Two cameras are mounted on the top, two on the right and another two cameras mounted on the left of the fruit. X-ray imaging is used for inspecting the biological defects. Image processing is used to analyze the fruit's features; size, color, shape and the grade is determined based on the features. The developed system is built from a combination of advanced designs, expert fabrications and automatic mechanical control. J. V. Frances, J. Calpe, E. Soria, M. Martinez, A. Rosado, A.J. Serrano, J. Calleja, M. Diaz [4] presented a procedure to improve the performance, whether increasing speed or accuracy, of the load-cell-based weighting subsystem in a fruit sorting and grading machine to achieve an accuracy of +1 gram. Wong Bing Yit, Nur Badariah Ahmad Mustafa, Zaipatimah Ali, Syed Khaleel Ahmed, Zainul Abidin Md Sharrif [5] proposed new MMS-based system design 
and developed with signal processing for fruit grading for consumers. The prototype network architecture, integration of wireless messaging system with signal processing between mobile consumers for development purposes was studied, proposed and designed.

\section{THE SYSTEM OVERVIEW}

This automated system is designed to overcome the problems of manual techniques. Here the hardware model is designed which contains conveyor system, grading assembly which contains three plates to which DC motor is connected, digital camera, IR sensor, LPC2138 processor, LCD display on field and grading assembly, The block diagram of a system is shown in Figure 1,

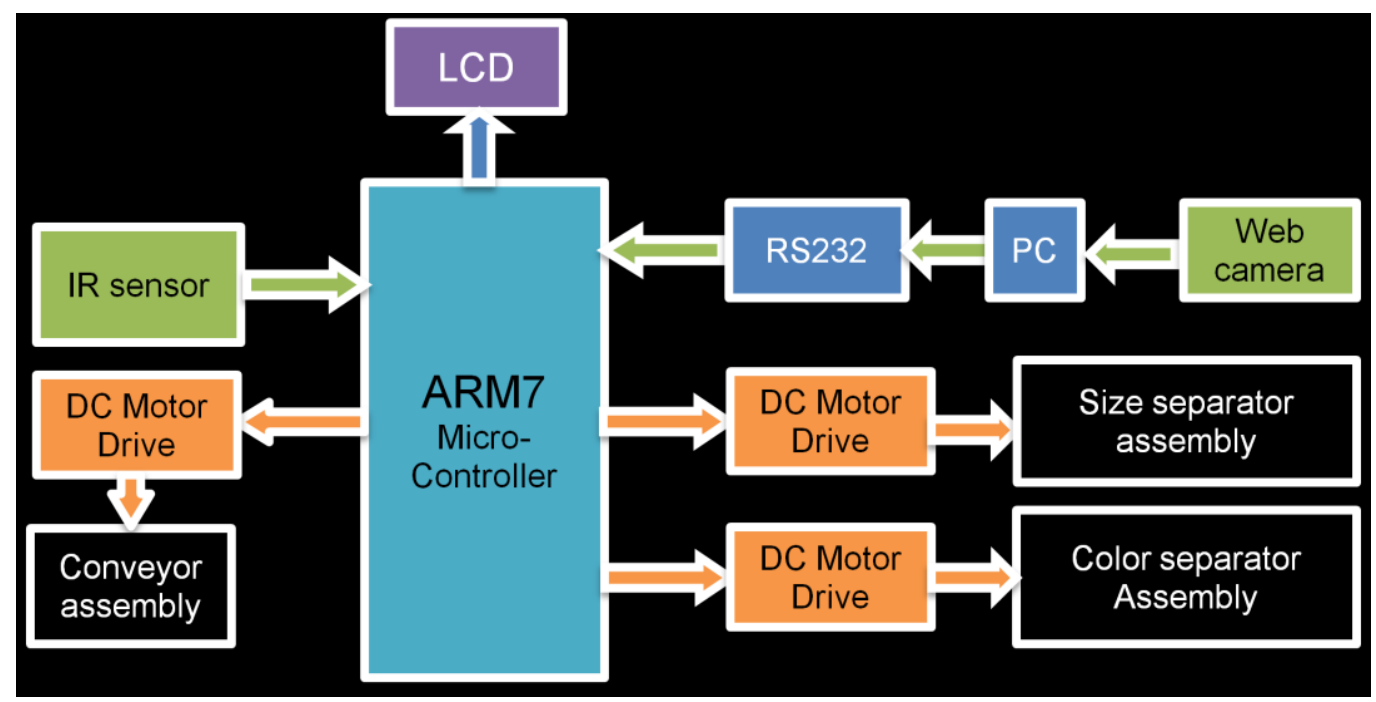

Figure 1: System Block Diagram

The image could be captured using a regular digital camera. Here we have used for capturing image the iball twist cam which is CMOS based camera[3]. The system arrangement is done as shown below the basic aim is to obtaining the fruit's features. The system consists of several steps like feature extraction, sorting and grading. As proposed in [1], to avoid shadow, two annular lights are used to supply well- distributed light.

The black background color in image is easier to extract the fruit edge characters later. So the background is set black in whole process of image capture. The light and camera location is as shown in Figure 2.

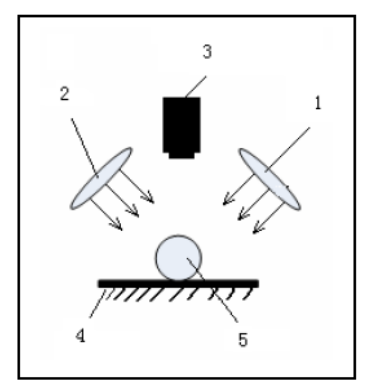

Figure 2:Fruit system light and camera location. 1-light; 2-light; 3-cmos camera; 4-conveyor belt; 5-fruit

For grading using first choice camera position is adjusted in such a way that for capturing live image of a fruit the camera is continuously scanning the conveyor belt in video mode, when conveyor stops as fruit is detected by IR system camera can capture top view image of fruit. The black background color in image is easier to extract the fruit edge characters later [1] so black color is used for the conveyor system. The captured image is given as an input to the MATLAB software which extracts (detects) color and size of a fruit, this data is transferred to ARM based system by using RS232 and com port and accordingly control action is taken place, later conveyor starts and Then fruit is collected in main plate of grading assembly. If fruit is red color (as detected by MATLAB) then the main plate is moving anticlockwise and accordingly the fruit is collected in lower plate 1, now depending on the size of fruit(as detected by MATLAB) it can be graded as a small or big fruit. If fruit is of big size the lower plate 1 will be moving anticlockwise and if fruit is of small size the lower plate 1 will be moving clockwise, Similarly, if fruit is of green color (as detected by MATLAB) then the main plate is moving clockwise and accordingly the fruit is collected in lower plate 2, now depending on the size of 
fruit (as detected by MATLAB) it can be graded as a small or big fruit. If fruit is of big size the lower plate 2 will be moving anticlockwise and if fruit is of big size the lower plate 2 will be moving clockwise.

\section{Fruit Size Detecting And Grading}

\section{A. Processing flow}

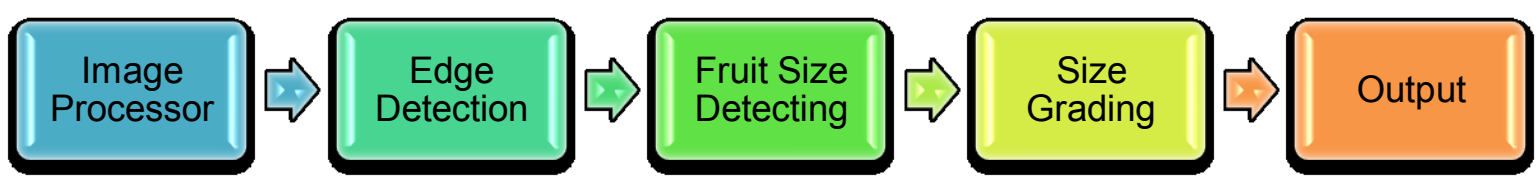

Figure 3: Fruit image size detecting and grading flow

Take apple as the processing example, according to [1],the apple size is its diameter, which is the longest distance in the apple's cross section. So the detecting program is focused on how to calculate the diameter in an apple side view image. The fruit image size detecting and grading processing flow is shown in Fig.3

\section{B. Color Detection}

In the process of fruit color is detected according toRGB values [5], here fruits are sorted according to color and size. So for e.g. two fruits are considered say tomato having red color and guava having green color, so in this step work is going to find out color of a fruit by using RGB values of an image taken from the camera, this image can be processed by using MATLAB software and accordingly color can be detected i.e. green or red.

Color detection algorithm:

1) Start

2) Read the input color image using imread function.

3) Read the input pixel of color image in three different planes (RGB) and store it into threevariable r, g, and b.

4) Read the small region of fruit to detect color of fruit.

5) Store in different variable r1, g1, b1.

6) Calculate the mean of $\mathrm{r} 1, \mathrm{~g} 1, \mathrm{~b} 1$ and store intovariable $\mathrm{r} 2, \mathrm{~g} 2, \mathrm{~b} 2$.

7) Compare the value with threshold.

8) If g2>threshold, Color detected is green.

9) If $\mathrm{r} 2>$ threshold, Color detected is Red.

10) End.

\section{Edge Detection}

Once color is detected, there is a need to find out size of a fruit. The size of circular shaped fruit is its diameter [1]. The edge extraction is key factor for size detecting. After gray image, the most powerful edgedetection method that finds edge is the canny method.

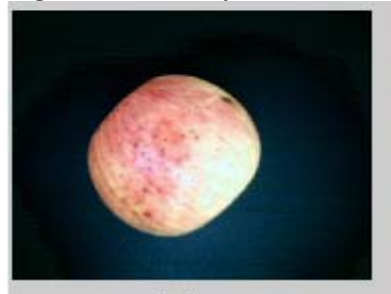

(a)

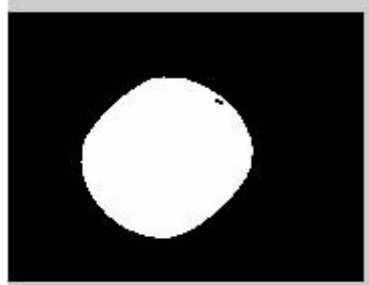

(c)

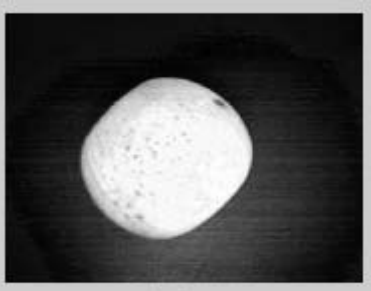

(b)

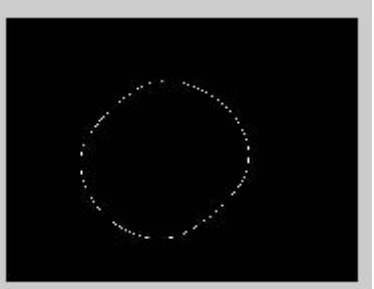

(d)

Figure 4: The processing fruit image. (a) the original image;(b)gray image;(c)diversion image;(d)tracking edge 
The Canny method differs from the other edge-detection methods [7] in that it uses two different thresholds (to detect strong and weak edges)and includes the weak edges in the output only if they are connected to strong edges. This method is therefore less likely than the others to be fooled by noise, and more likely to detect true weakedges.

\section{Fruit Size detecting algorithm:}

In order to calculate this diameter, the fruit's natural symmetry is considered, so the fruit size detecting algorithm based on its symmetry mainly contains two parts: finding the center coordinates of fruit's shape in image and finding fruit's axis in image [7]. The algorithm is described as follows:

1) Finding the center coordinate of fruit's shape in image: The center coordinate can be easily calculated once finding the edge sequence points. Suppose the finding edge sequence point is $q\left(x_{i}, y_{i}\right), i=1, n$. the center coordinates of fruit's shape is $\left(c_{x}, c_{y}\right)$, it can be calculated by (1) and (2) as in [1]:

$$
\begin{gathered}
\mathrm{c}_{\mathrm{x}}=\frac{\sum_{\mathrm{k}=1}^{\mathrm{n}}\left[\mathrm{y}_{\mathrm{k}}\left(\mathrm{x}_{\mathrm{k}}^{2}-\mathrm{x}_{\mathrm{k}-1}^{2}\right)-\mathrm{x}_{\mathrm{k}}^{2}\left(\mathrm{y}_{\mathrm{k}}-\mathrm{y}_{\mathrm{k}-1}\right)\right]}{2 \sum_{\mathrm{k}=1}^{\mathrm{n}}\left[\mathrm{y}_{\mathrm{k}}\left(\mathrm{x}_{\mathrm{k}}-\mathrm{x}_{\mathrm{k}-1}\right)-\left(\mathrm{y}_{\mathrm{k}}-\mathrm{y}_{\mathrm{k}-1}\right)\right]} \\
\mathrm{c}_{\mathrm{y}}=\frac{\sum_{\mathrm{k}=1}^{\mathrm{n}}\left[\mathrm{y}_{\mathrm{k}}^{2}\left(\mathrm{x}_{\mathrm{k}}-\mathrm{x}_{\mathrm{k}-1}\right)-\mathrm{x}_{\mathrm{k}}\left(\mathrm{y}_{\mathrm{k}}^{2}-\mathrm{y}_{\mathrm{k}-1}^{2}\right)\right]}{2 \sum_{\mathrm{k}=1}^{\mathrm{n}}\left[\mathrm{y}_{\mathrm{k}}\left(\mathrm{x}_{\mathrm{k}}-\mathrm{x}_{\mathrm{k}-1}\right)-\mathrm{x}_{\mathrm{k}}\left(\mathrm{y}_{\mathrm{k}}-\mathrm{y}_{\mathrm{k}-1}\right)\right]}
\end{gathered}
$$

2) Finding the fruit's axis in image:

After get the center coordinates of fruit's shape in image, the diameter sequence from the edge point to the center can be also acquired, that is $p(j) j=1, \ldots, n$. and then it's even points selected from $p(j)$ called

$\mathrm{r}(\mathrm{j}), \mathrm{j}=1, \ldots, \mathrm{m}$. suppose $\mathrm{h} \square 1, \ldots, \mathrm{m} / 2$. So the $\mathrm{r}(\mathrm{j})$ can be divided in two parts by $\mathrm{h}$, and then calculating the $\mathrm{g}$, which is described by (3).

$$
\begin{gathered}
g=\sum_{l=1}^{m / 2}|r(h+l)-r(h-l)|(\mathrm{h}=1,2, \ldots, \mathrm{m} / 2) \\
\text { If }|\mathrm{h}-1| \leq 1, \mathrm{r}(\mathrm{h}-1)=\mathrm{r}(\mathrm{m}+1-\mathrm{h}-1)
\end{gathered}
$$

The direction of $r(h)$ is the fruit's axis in image while $g$ the getting its minimum. Following the below method, the fruit's axis point and center point is found in image as shown in Figure. 5. Once known the axis point and the center point, a line through the center point which is vertical to the line from axis point to center point will be crossed with the edge sequences, two edge points that on the line will be searched. Suppose the two points is $\left(\mathrm{x}_{1}, \mathrm{y}_{1}\right)$ and $\left(\mathrm{x}_{2}, \mathrm{y}_{2}\right)$ in order to improving the system's speed, the diameter is calculated by (4) indicates the fruit's real maximal diameter in image. From the detecting result in Figure 5,

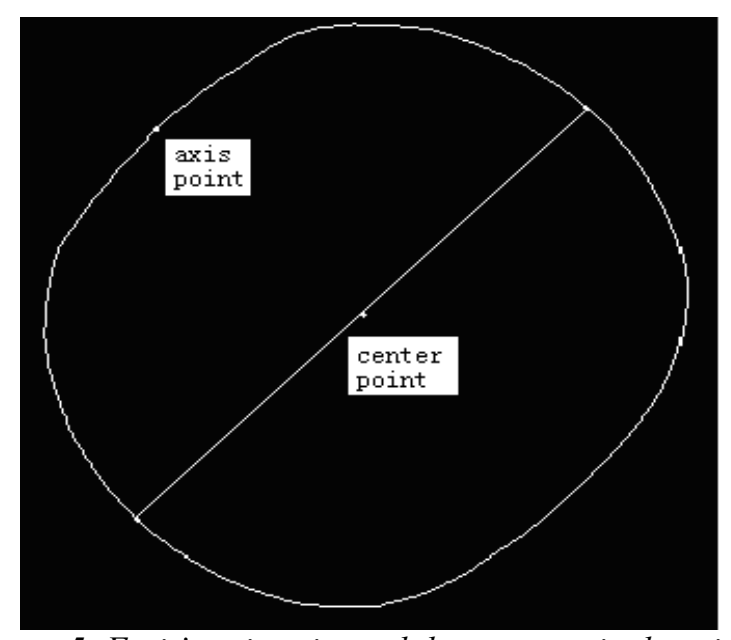

Figure 5: Fruit's axis point and the center point location 1

This method can find the axis point accurately in a fruit image. And also, it still can find the two points while the fruit's location changed. So this method can satisfy the fruit size detecting on line which its location changed often.

$$
d=\left[\left(x_{1}-x_{2}\right)^{2}+\left(y_{1}-y_{2}\right)^{2}\right]^{1 / 2}
$$




\section{E. Fruit Size Grading}

According to apple state criterion, size grading is judged by the detected diameter of an apple, [6] the criterion is shown as the table 1.

TABLE 1. Apple Size Grading Criterion (mm:Millimeter)

\begin{tabular}{|c|c|}
\hline Criteria & Diameter \\
\hline Big & $\geq 60 \mathrm{~mm}$ \\
\hline Small & $\leq 50 \mathrm{~mm}$ \\
\hline
\end{tabular}

\section{CONCLUSION}

The proposed system is a demo version, so for a large scale production the number of cameras and length of conveyor system can be modified. This work presents new integrated techniques for sorting and grading of different fruits. Generally image capture is a big challenge as there is a chance of high uncertainty due to the external lighting conditions, so we are taking the advantage of gray scale image which are less effected to the external environment changes as well as beneficial for finding size of a fruit. Same way while collecting fruit from conveyor system by a main plate there is variation in the weight measurement of a fruit so further design can be modified so fruits can be collected stably. Speed and efficiency of a system can be further improved by using ARM9 or ARM11 processor for the same purpose.

\section{REFERENCES}

[1] Hongshe Dang, Jinguo Song, Qin Guo, “A Fruit Size Detecting and Grading System Based on Image Processing,” 2010 Second International Conference on Intelligent Human-Machine Systems and Cybernetics,pp83-86.

[2] Harshavardhan G. Naganur, Sanjeev S. Sannakki, Vijay SRajpurohit, Arunkumar R, “Fruits Sorting and Grading usingFuzzy Logic," International Journal of Advanced Research inComputer Engineering \& Technology (IJARCET) Volume 1,Issue 6, August 2012,pp 117-122.

[3] John B. Njoroge. Kazunori Ninomiya. Naoshi Kondo andHideki Toita, “Automated Fruit Grading System using ImageProcessing," The Society of Instrument and Control Engineers(SICE2002), Osaka, Japan, August 2002, pp 1346-1351.

[4] J. V. Frances, J. Calpe, E. Soria, M. Martinez, A. Rosado, A.J.Serrano, J. Calleja, M. Diaz, "Application of ARMA modeling to the improvement of weight estimations in fruit sorting and grading machinery," IEEE 2000, pp 3666-3669

[5] Wong Bing Yit, Nur Badariah Ahmad Mustafa, ZaipatimahAli, Syed Khaleel Ahmed, Zainul Abidin Md Sharrif, "Design and Development of a Fully Automated Consumer-based Wireless Communication System For Fruit Grading”, ISCIT 2009 , pp $364-$ 369 .

[6] Naoshi Kondo, "Fruit Grading Robot", Proceedings of the2003 IEEE/ASME International Conference on AdvancedIntelligent Mechatronics (AIM 2003), pp 1366-1371.

[7] R. C. Gonzalez, R. E. Woods," Digital Image Processing", Pearson Education.IIEd.,2002 